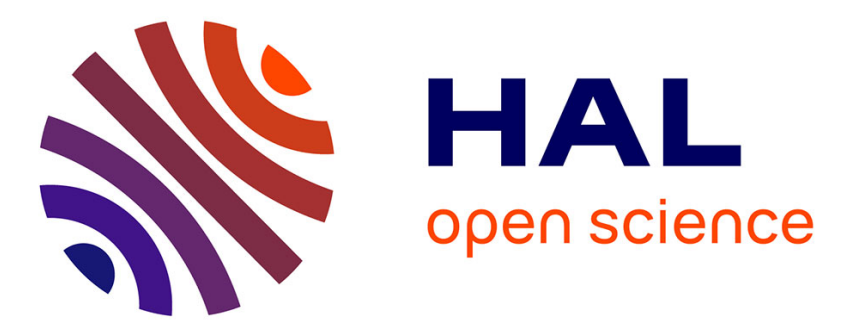

\title{
Quasiparticle kinetic theory for Calogero models
}

Vir Bannerjee Bulchandani, Manas Kulkarni, Joel E Moore, Xiangyu Cao

\section{To cite this version:}

Vir Bannerjee Bulchandani, Manas Kulkarni, Joel E Moore, Xiangyu Cao. Quasiparticle kinetic theory for Calogero models. Journal of Physics A: Mathematical and Theoretical, 2021,54 (47), 10.1088/1751- . hal-03383709

\section{HAL Id: hal-03383709 \\ https: / hal.sorbonne-universite.fr/hal-03383709}

Submitted on 18 Oct 2021

HAL is a multi-disciplinary open access archive for the deposit and dissemination of scientific research documents, whether they are published or not. The documents may come from teaching and research institutions in France or abroad, or from public or private research centers.
L'archive ouverte pluridisciplinaire HAL, est destinée au dépôt et à la diffusion de documents scientifiques de niveau recherche, publiés ou non, émanant des établissements d'enseignement et de recherche français ou étrangers, des laboratoires publics ou privés. 
ACCEPTED MANUSCRIPT

\section{Quasiparticle kinetic theory for Calogero models}

To cite this article before publication: Vir Bannerjee Bulchandani et al $2021 \mathrm{~J}$. Phys. A: Math. Theor. in press https://doi.org/10.1088/1751$\underline{8121 / \mathrm{ac} 2 f 8 \mathrm{e}}$

\section{Manuscript version: Accepted Manuscript}

Accepted Manuscript is "the version of the article accepted for publication including all changes made as a result of the peer review process, and which may also include the addition to the article by IOP Publishing of a header, an article ID, a cover sheet and/or an 'Accepted Manuscript' watermark, but excluding any other editing, typesetting or other changes made by IOP Publishing and/or its licensors"

This Accepted Manuscript is @ 2021 IOP Publishing Ltd.

During the embargo period (the 12 month period from the publication of the Version of Record of this article), the Accepted Manuscript is fully protected by copyright and cannot be reused or reposted elsewhere.

As the Version of Record of this article is going to be / has been published on a subscription basis, this Accepted Manuscript is available for reuse under a CC BY-NC-ND 3.0 licence after the 12 month embargo period.

After the embargo period, everyone is permitted to use copy and redistribute this article for non-commercial purposes only, provided that they adhere to all the terms of the licence https://creativecommons.org/licences/by-nc-nd/3.0

Although reasonable endeavours have been taken to obtain all necessary permissions from third parties to include their copyrighted content within this article, their full citation and copyright line may not be present in this Accepted Manuscript version. Before using any content from this article, please refer to the Version of Record on IOPscience once published for full citation and copyright details, as permissions will likely be required. All third party content is fully copyright protected, unless specifically stated otherwise in the figure caption in the Version of Record.

View the article online for updates and enhancements. 


\title{
Quasiparticle kinetic theory for Calogero models
}

\author{
Vir B. Bulchandani, ${ }^{1}$ Manas Kulkarni, ${ }^{2}$ Joel E. Moore,,${ }^{3,4}$ and Xiangyu Cao ${ }^{5}$ \\ ${ }^{1}$ Princeton Center for Theoretical Science, Princeton University, Princeton, New Jersey 08544, USA \\ ${ }^{2}$ International Centre for Theoretical Sciences, Tata Institute of Fundamental Research, Bengaluru 560089, India \\ ${ }^{3}$ Department of Physics, University of California, Berkeley, California 94720 USA \\ ${ }^{4}$ Materials Sciences Division, Lawrence Berkeley National Laboratory, Berkeley, California 94720, USA \\ ${ }^{5}$ Laboratoire de Physique de l'Ecole Normale Supérieure, ENS, Université PSL, \\ CNRS, Sorbonne Université, Université de Paris, 75005 Paris, France
}

(Dated: October 4, 2021)

We show that the quasiparticle kinetic theory for quantum and classical Calogero models reduces to the free-streaming Boltzmann equation. We reconcile this simple emergent behaviour with the strongly interacting character of the model by developing a Bethe-Lax correspondence in the classical case. This demonstrates explicitly that the freely propagating degrees of freedom are not bare particles, but rather quasiparticles corresponding to eigenvectors of the Lax matrix. We apply the resulting kinetic theory to classical Calogero particles in external trapping potentials and find excellent agreement with numerical simulations in all cases, both for harmonic traps that preserve integrability and exhibit perfect revivals, and for anharmonic traps that break microscopic integrability. Our framework also yields a simple description of multi-soliton solutions in a harmonic trap, with solitons corresponding to sharp peaks in the quasiparticle density. Extensions to quantum systems of Calogero particles are discussed.

\section{INTRODUCTION}

The emergence of macroscopic fluid behaviour from the chaotic motion of microscopic particles is a truth universally acknowledged, whose mathematical derivation nevertheless continues to present insurmountable difficulties. Aside from certain tractable classical cases ${ }^{1}$ and a handful of quantum examples ${ }^{2}$, microscopic derivations of hydrodynamics remain beyond reach at present. A significant conceptual advance in this area was the realization that large-scale dynamics in a wide range of extended integrable systems ${ }^{3 / 4}$ could be reduced to a kinetic theory of solitons $5+12$. This synthesis has created a powerful and increasingly rigorous ${ }^{13}$ to tool for understanding the emergence of hydrodynamic behaviour in realistic models from microseopic first principles, that moreover yields excellent agreement with stateof-the-art experimental results $17+19$.

One experimentally important question that remains unclear, despite several recent related studies 20 25, is the extent to which a kinetic theory of solitons continues to provide an accurate description in the presence of integrabilitybreaking trapping potentials. From this viewpoint, the family of Calogero-type models, which are integrable onedimensional systems that remain integrable in the presence of certain carefully chosen trapping potentials 26 , are natural objects of study. Moreover, their (zero temperature) hydrodynamics has been explored in some depth 27.30 .

In this paper, we develop a quasiparticle kinetic theory for Calogero models. We first derive the kinetic theory of the quantum Calogero model using established thermodynamic Bethe ansatz techniques ${ }^{3 / 4}$, showing that it reduces to a free-streaming Boltzmann equation. We then obtain the kinetic theory of the classical Calogero model as a semiclassical limit of the kinetic theory of the quantum model, following the analogous procedure for the Toda lattic ${ }^{31} 33$ (see also Refs. 34 38), which also turns out to be of non-interacting Boltzmann form. For the classical Calogero model, we explain this simple behaviour using the Bethe-Lax correspondence proposed in previous work ${ }^{31}$. This provides an independent check on the kinetic theory description and illustrates that the freely streaming degrees of freedom are not bare particles, as one might expect from the absence of velocity dressing, but instead quasiparticles that can be identified with eigenvectors of the Lax matrix.

We then study the validity of the quasiparticle kinetic theory, as augmented by a naïve Boltzmann force term, in the presence of external trapping potentials. For the integrability-preserving case of a harmonic trapping potential (also known as the Calogero-Moser model) we find that this kinetic theory captures the finite-temperature dynamics to within numerical accuracy, including non-trivial features such as perfect revivals and soliton excitations 30 . For integrability-breaking anharmonic potentials, we again find excellent agreement with numerics. This is attributed to an unusual robustness of integrability of the Calogero dynamics to external trapping potentials. We close with some remarks on the dynamics of trapped quantum Calogero particles. 


\section{KINETIC THEORY}

\section{A. Quantum Calogero model}

Consider the $N$-particle, quantum (rational) Calogero model, with Hamiltonian

$$
H=\sum_{i=1}^{N}-\frac{1}{2} \partial_{i}^{2}+\sum_{i<j} \frac{g}{\left(x_{i}-x_{j}\right)^{2}},
$$

where we set $\hbar=m=1$ and $g=\alpha(\alpha-1)$ for some $\alpha>0$. This model is integrable, and its exact spectrum can be obtained from Sutherland's method of asymptotic Bethe ansatz ${ }^{39}$. The two-particle phase shift is given by

$$
\varphi(k)=\pi(\alpha-1) \operatorname{sgn}(k),
$$

implying that the differential phase shift is

$$
K_{k, k^{\prime}}=\frac{1}{2 \pi} \varphi^{\prime}\left(k-k^{\prime}\right)=(\alpha-1) \delta\left(k-k^{\prime}\right) .
$$

For states in thermal equilibrium with chemical potential $\mu$ and inverse temperature $\beta$, it follows by thermodynamic Bethe ansatz ${ }^{40}$ that quasiparticle energies satisfy the Yang-Yang equation

$$
\epsilon_{k}=\frac{k^{2}}{2}-\mu+\frac{1}{\beta} \int_{-\infty}^{\infty} d k^{\prime} K_{k, k^{\prime}} \ln \left(1+e^{-\beta \epsilon_{k^{\prime}}}\right),
$$

while the total density of states satisfies

$$
\rho_{k}^{t}+\int_{-\infty}^{\infty} d k^{\prime} K_{k, k^{\prime}} \theta_{k^{\prime}} \rho_{k^{\prime}}^{t}=\frac{1}{2 \pi},
$$

with Fermi factors given by $\theta_{k}=\left(1+e^{\beta \epsilon_{k}}\right)^{-1}$. For the phase shift Eq. (3), different values of pseudomomentum $k$ decouple. The Yang-Yang equation can thus be expressed as a transcendental equation for $\theta_{k}$, that fixes the occupied density of states $\rho_{k}=\rho_{k}^{t} \theta_{k}$ :

$$
\begin{aligned}
\frac{\theta_{k}}{\theta_{k}+\left(1-\theta_{k}\right)^{\alpha}} & =\frac{1}{1+e^{\beta\left(k^{2} / 2-\mu\right)}}, \\
\rho_{k} & =\frac{1}{2 \pi} \frac{\theta_{k}}{1+(\alpha-1) \theta_{k}} .
\end{aligned}
$$

For $\alpha=0$, Eq. (7) recovers the occupation numbers for free bosons, while for $\alpha=1$, it yields the occupation numbers for free fermions. For other values of $\alpha$, these equations describe the thermodynamics of free anyons 41 .

To proceed from thermodynamic Bethe ansatz to the generalized hydrodynamics of the Calogero model, we require an expression for the quasiparticle group velocity on a given equilibrium state ${ }^{314}$. (Note that for the Calogero model, the kinetic theory description is expected to be equivalent to a generalized hydrodynamics consisting of countably many hydrodynamic equations, with no subtleties arising due to vacuum modes ${ }^{42}$.) Recall that the derivatives of energy and momentum for a quasiparticle excitation on a given equilibrium state with Fermi factors $\left\{\theta_{k}\right\}_{k \in \mathbb{R}}$ satisfy

$$
\begin{array}{r}
\epsilon_{k}^{\prime}+\int_{-\infty}^{\infty} d k^{\prime} K_{k, k^{\prime}} \theta_{k^{\prime}} \epsilon_{k^{\prime}}^{\prime}=k, \\
p_{k}^{\mathrm{dr}{ }_{k}^{\prime}}+\int_{-\infty}^{\infty} d k^{\prime} K_{k, k^{\prime}} \theta_{k^{\prime}} p_{k^{\prime}}^{\mathrm{dr}^{\prime}}=1 .
\end{array}
$$

For the differential phase shift Eq. (3), these reduce to

$$
\begin{aligned}
\left(1+(\alpha-1) \theta_{k}\right) \epsilon_{k}^{\prime} & =k, \\
\left(1+(\alpha-1) \theta_{k}\right) p^{\mathrm{dr}^{\prime}} & =1 .
\end{aligned}
$$

It follows that the quasiparticle group velocity in a given equilibrium state simply equals the bare velocity:

$$
v_{k}=\epsilon_{k}^{\prime} / p^{\mathrm{dr}}{ }_{k}^{\prime}=k .
$$


Thus the Bethe-Boltzmann equation for the Calogero model reduces to the free-streaming Boltzmann equation,

$$
\partial_{t} \rho_{k}+k \partial_{x} \rho_{k}=0 \text {. }
$$

Note that state independence of the group velocity Eq. (11) additionally implies that its fluctuations vanish on equilibrium states, and hence that the Navier-Stokes correction to Eq. 12 vanishes $\frac{43 \mid 44}{4}$. This implies that the Calogero model is non-interacting in the sense of Spohn $\frac{45}{\text {, }}$, despite its singular inter-particle interactions.

\section{B. Classical Calogero model}

We now derive the thermodynamic Bethe ansatz and kinetic theory for the classical Calogero model by passing to the semiclassical limit, adapting the analogous derivation for the Toda lattice 31 33. Our starting point is the quantum Calogero model with Planck's constant restored, namely

$$
H=\sum_{i=1}^{N}-\frac{1}{2}\left(\hbar \partial_{i}\right)^{2}+\sum_{i<j} \frac{\hbar^{2} \alpha(\alpha-1)}{\left(x_{i}-x_{j}\right)^{2}}
$$

The semiclassical limit is obtained by expressing the quantum TBA equations in terms of classical momentum $p=\hbar k$, before taking $\hbar \rightarrow 0$ with $\ell=\hbar \alpha$ constant 46 . To obtain a finite free energy in this limit, we define classical energies and chemical potentials

$$
\epsilon_{p}^{c l}=\epsilon_{p}+\frac{1}{\beta} \ln \hbar / \ell, \quad \mu^{c l}=\mu-\frac{1}{\beta} \ln \hbar / \ell .
$$

For non-zero $\hbar$, the Yang-Yang equation and dressing equations can be written exactly in terms of $p$, as 31

$$
\epsilon_{p}^{c l}=\frac{p^{2}}{2}-\mu^{c l}+\frac{1}{\beta \hbar} \int_{-\infty}^{\infty} d p^{\prime} K_{p / \hbar, p^{\prime} / \hbar} \ln \left(1+\hbar \ell^{-1} e^{-\beta \epsilon_{p^{\prime}}^{c l}}\right)
$$

and

$$
\begin{aligned}
& \epsilon_{p}^{c l^{\prime}}+\int_{-\infty}^{\infty} d p^{\prime} K_{p / \hbar, p^{\prime} / \hbar}\left(\theta_{p^{\prime}} / \hbar\right) \epsilon_{p^{\prime}}^{c l^{\prime}}=p, \\
& p_{p}^{\mathrm{dr}_{p}^{\prime}}+\int_{-\infty}^{\infty} d p^{\prime} K_{p / \hbar, p^{\prime} / \hbar}\left(\theta_{p^{\prime}} / \hbar\right) p_{p^{\prime}}^{\mathrm{dr}^{\prime}}=1 .
\end{aligned}
$$

The classical total density of states satisfies $2 \pi \hbar \tilde{\rho}_{p}^{t}=p^{\mathrm{dr}}{ }_{p}$. In the limit $\hbar \rightarrow 0$, the phase shift is given by

$$
K_{p, p^{\prime}}^{c l}=\lim _{\hbar \rightarrow 0} K_{p / \hbar, p^{\prime} / \hbar}=\lim _{\hbar \rightarrow 0} \hbar(\alpha-1) \delta\left(p-p^{\prime}\right)=\ell \delta\left(p-p^{\prime}\right),
$$

and the Yang-Yang equation becomes

$$
\epsilon_{p}^{c l}=\frac{p^{2}}{2 m}-\mu^{c l}+\frac{1}{\beta} e^{-\beta \epsilon_{p}^{c l}} .
$$

Note that this is independent of the interaction strength, $\ell$. In fact, so are the dressing equations, and read

$$
\begin{gathered}
\left(1+e^{-\beta \epsilon_{p}^{c l}}\right) \epsilon_{p}^{c l^{\prime}}=p, \\
\left(1+e^{-\beta \epsilon_{p}^{c l}}\right) p_{p}^{\mathrm{dr}^{\prime}}=1 .
\end{gathered}
$$

This reflects the fact that the leading asymptotic kinematics in the classical Calogero mode $\sqrt{47}$ is independent of $\ell$. However, the classical density of occupied states does depend on $\ell$, and is given by

$$
\widetilde{\rho}_{p}=\lim _{\hbar \rightarrow 0} \widetilde{\rho}_{p}^{t} \theta_{p}=\frac{1}{2 \pi} \frac{1}{1+e^{\beta \epsilon_{p}^{c l}}} \frac{1}{\ell} .
$$

Finally, we note that the classical dressing equations Eqs. 20 and 21 imply an effective velocity

$$
v_{p}=\epsilon_{p}^{c l^{\prime}} / p_{p}^{\mathrm{dr}}{ }_{p}^{\prime}=p
$$


that is free-particle like. The resulting kinetic theory takes the form of a free-streaming Boltzmann equation,

$$
\partial_{t} \widetilde{\rho}_{p}+p \partial_{x} \widetilde{\rho}_{p}=0
$$

as in the quantum case.

In the presence of an external trapping potential, the naïve modification of Eq. (24) by a Boltzmann force term reads

$$
\partial_{t} \widetilde{\rho}_{p}+p \partial_{x} \widetilde{\rho}_{p}-V^{\prime}(x) \partial_{p} \widetilde{\rho}_{p}=0 .
$$

The regime of validity of this equation is not immediately clear, as the presence of an external trapping potential is expected in general to break integrability and generate finite quasiparticle lifetimes, ultimately invalidating any description based on a kinetic theory of quasiparticles. This crossover time-scale for the onset of chaos, which depends on the initial condition, was previously obtained for the classical hard rod model with integrability broken by a harmonic trap 21 .

However, Calogero-type models are unusual among integrable models because they remain integrable in the presence of certain carefully chosen external trapping potentials. For the rational Calogero model considered here, the known integrability-preserving potentials take the form 4

$$
V(x)=a x^{4}+b x^{3}+c x^{2}+d, \quad a, b, c, d \in \mathbb{R} .
$$

For this restricted class of potentials, the validity of Eq. 25) is more plausible. This result also suggests a qualitative robustness of Calogero particles to integrability-breaking by generic smooth potentials $V(x)$, since approximating $V(x)$ by the first four terms of a Taylor expansion about any given point $x=x_{0}$ will always yield an integrable model.

\section{BETHE-LAX CORRESPONDENCE}

The classical Calogero model exhibits a Lax pair formalism ${ }^{48}$, just as for the classical Toda mode $\frac{49}{4}$. In previous work, it was noted that conserved quasiparticles in the Toda model could be understood as eigenvectors of the Lax

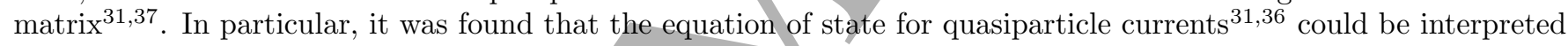
as a statement about thermal averages of eigenvectors of the Lax matrix. It is natural to conjecture that a similar correspondence holds for the classical Calogero model. We shall show that this is indeed the case, and moreover, that the Bethe-Lax correspondence explains why the current density of states takes the simple, non-interacting form

$$
\widetilde{\rho}_{p}^{J}=p \widetilde{\rho}_{p} .
$$

\section{A. From matrix models to Lax pairs: a reminder}

It will be useful to recall that the classical Calogero model can be obtained from the Hamiltonian reduction of a Hermitian matrix model $\sqrt[48]{ }$, defined by the Lagrangian

$$
\mathcal{L}=\frac{1}{2} \operatorname{Tr}\left(\dot{M} \dot{M}^{\dagger}\right)-\operatorname{Tr} V(M),
$$

where $M$ is an $N \times N$ Hermitian matrix and $V(M)$ is usually a polynomial function (the potential). The equations of motion read

where $F(x)=-V^{\prime}(x)$ is the force.

$$
\frac{d M}{d t}=\Lambda, \frac{d \Lambda}{d t}=F(M)
$$

The $S U(N)$ symmetry of the action means that one should be able to describe the dynamics solely in terms of the eigenvalues of $M$. To this end, let $U$ denote the dynamical unitary transformation diagonalizing $M$ :

$$
M=U^{\dagger} X U, X=\operatorname{diag}\left(x_{1}, \ldots, x_{N}\right) .
$$

Then the motion of $M$ has a "radial" and "angular" part

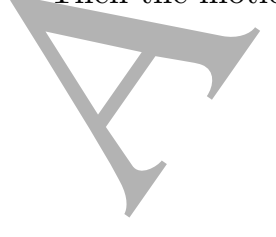

$$
\frac{d}{d t} M=U^{\dagger}(\dot{X}+i[X, A]) U, i A:=\dot{U} U^{\dagger} .
$$


where $A$ is the Hermitian matrix generating the angular motion. Letting $L$ denote the rotated version of $\Lambda$,

$$
\Lambda=U^{\dagger} L U, \frac{d}{d t} \Lambda=U^{\dagger}(\dot{L}+i[L, A]) U
$$

the equations of motion for $M$ and $\Lambda$ can be rewritten in terms of $X$ and $L$ as

$$
\begin{aligned}
\dot{X}+i[X, A] & =L, \\
\dot{L}+i[L, A] & =F(X)
\end{aligned}
$$

(note that we used $U F(M) U^{\dagger}=F(X)$, which assumes that $F(M)$ is analytic). To see how the Calogero model emerges explicitly, we need to fix the "angular momentum" associated with the $S U\left(N^{*}\right)$ symmetry. The conserved current is

$$
J=-i[M, \dot{M}] \Rightarrow \dot{J}=0 .
$$

The Calogero model comes from choosing a traceless matrix with one-dimensional kernel for $J$ :

$$
J=\ell\left(v v^{\dagger}-I\right), v^{\dagger} v=N
$$

Then, since

$$
J=-i[M, \dot{M}]=U^{\dagger}[X,[X, A]] U
$$

we have

$$
[X,[X, A]]=U J U^{\dagger}=\ell\left(u u^{\dagger}-I\right), u:=U v .
$$

Now the LHS has zero diagonal elements, so $\left|u_{i}\right|^{2}=1$, and we can set $u_{i}=1$. Then the above equation implies $A_{i j}=\ell x_{i j}^{-2}$ for $i \neq j\left(x_{i j}:=x_{i}-x_{j}\right)$. The diagonal elements are set by $u^{\dagger} A=0$, so that we have

$$
A_{i j}=-\delta_{i j} \ell \sum_{k \neq i} x_{i k}^{-2}+\left(1-\delta_{i j}\right) \ell x_{i j}^{-2} .
$$

It follows from 33 that

$$
L_{i j}=\delta_{i j} \dot{x}_{i}+\left(1-\delta_{i j}\right) i \ell x_{i j}^{-1}
$$

We have thus recovered the Lax pair for the classical Calogero model.

\section{B. Statement of the correspondence}

In the absence of an external potential, the force $F(X)$ vanishes and the flow of the Lax matrix is isospectral, i.e. its eigenvalues are conserved. The question then arises of how these conserved eigenvalues relate to the conserved quasiparticle momenta in the classical Boltzmann description, Eq. 24. As in previous work on the Toda lattice ${ }^{31}$, we argue that these quantities can be identified, and moreover that the eigenvectors of the Lax matrix are naturally interpreted as quasiparticles, giving rise to a "Bethe-Lax correspondence" between the semiclassical Bethe ansatz and the spectral data of the classical Lax matrix.

Concretely, note that if $V(M)=0$ in Eq. $(29)$, then $\dot{M}=\Lambda$ is conserved and by $S U(N)$ symmetry can be chosen to be diagonal:

$$
\Lambda=\operatorname{diag}\left(\lambda_{1}, \ldots, \lambda_{N}\right), \dot{\Lambda}=0 .
$$

Since $L$ and $\Lambda$ are related by the similarity transformation Eq. $(32)$, it follows that $\left\{\lambda_{i}\right\}_{i=1}^{N}$ are the eigenvalues of $L$. Also, since $L=U \Lambda U^{\dagger}$, we can directly identify the columns of $U$ with the eigenvectors of $L$, yielding the expressions

$$
U=\sum_{j}\left|\lambda_{j}\right\rangle\left\langle j|, L| \lambda_{j}\right\rangle=\lambda_{j}\left|\lambda_{j}\right\rangle, \frac{d}{d t}\left|\lambda_{j}\right\rangle=i A\left|\lambda_{j}\right\rangle,
$$

where the last identity follows from (31), as the "Schrödinger equation" corresponding to the unitary evolution $U$. It is also useful to define the spectrum of $X$ as $X\left|x_{a}\right\rangle=x_{a}\left|x_{a}\right\rangle$. 
We now claim that $\left|\lambda_{i}\right\rangle$ corresponds to a quasiparticle with momentum $\lambda_{i}$, which is delocalized in space as the "wavefunction" $\left|\lambda_{i}\right\rangle$, i.e., has a distribution with weight $\left|\left\langle x_{a} \mid \lambda_{i}\right\rangle\right|^{2}$ at $x_{a}$. Equivalently, we identify the density $\rho_{p}(x)$ with the local density of states of $L$ (which we shall call the empirical quasiparticle density) in the-hydrodynamic limit:

$$
\begin{aligned}
& \rho_{p}^{\mathrm{emp}}(x) \longrightarrow \rho_{p}(x) \text { in the hydrodynamic limit, where } \\
& \rho_{p}^{\mathrm{emp}}(x):=\sum_{a=1}^{N} \sum_{j=1}^{N}\left|\left\langle x_{a} \mid \lambda_{j}\right\rangle\right|^{2} \delta\left(x-x_{a}\right) \delta\left(p-\lambda_{j}\right) .
\end{aligned}
$$

Eq. (43) is the main result of this paper: it relates a microscopic configuration of the Calogero model to a macroscopic description in terms of the quasiparticle density. In what follows, we shall justify it with both analytical and numerical arguments.

\section{Relation to thermodynamic Bethe Ansatz}

A first consequence of the Bethe-Lax correspondence (44) is that the density of states of the Lax matrix, averaged over thermal configurations, is given by the solution of the classical Yang-Yang equation, see Eqs. (19) and 222):

$$
\widetilde{\rho}_{p}=\lim _{\substack{N, L \rightarrow \infty, N / L=\text { const }}} \frac{1}{L} \sum_{j=1}^{N} \delta\left(p-\lambda_{j}\right)
$$

where the bars denote thermal averages and the particles are assumed to be confined to a large box of length $L$ (this is necessary to define a thermal state, since otherwise the particles are unbounded ${ }^{48}$ ).

Eq. 45) can be checked analytically at zero temperature, at which the particles are immobile $\left(\dot{x}_{a}=0\right)$ and form a lattice, $x_{a}-x_{b}=(a-b) / \rho$, where $\rho=N / L$ is the density. In the $N \rightarrow \infty$ limit, the Lax matrix is a circulant matrix and can be diagonalized by Bloch plane waves in the Brillouin zone $[-\pi \rho, \pi \rho)$. As a result, we obtain a constant density of states in the interval $[-\ell \rho \pi, \ell \rho \pi]$, in agreement with the prediction of Eq. (22) (with $\beta \rightarrow \infty$ and suitable chemical potential).

At non-zero temperature, a proof of (45), as was achieved for the particle density of states for the Toda lattice by analogy with Dumitriu-Edelman random matrix ensembles 35 , seems beyond reach at present. Nevertheless, we can check this equation numerically by generating thermal states from classical Monte Carlo simulations and diagonalizing the $L$ matrix. We observe good agreement with numerics at various non-zero temperatures and densities. See Fig. 1 for detailed methods and a sample of results.

\section{Boltzmann equation}

A further important check of the Bethe-Lax correspondence is to show that the empirical quasiparticle density $\rho_{p}^{\mathrm{emp}}(x)$ (44) satisfies the Boltzmann equation (25), subject to an appropriate approximation in the hydrodynamic limit.

To this end, we rewrite the empirical quasiparticle density as

$$
\rho_{p}^{\mathrm{emp}}(x)=\operatorname{Tr}[\delta(p-L) \delta(x-X)], \text { where } \delta(p-L)=\frac{1}{2 \pi i}\left[(p-i \epsilon-L)^{-1}-(p+i \epsilon-L)^{-1}\right]_{\epsilon \rightarrow 0_{+}},
$$

and similarly for $\delta(x-X)$, by the Stieltjes inversion formula. Expanding the matrices inverses at complex infinity, the above formula can be viewed as a resummation of the moments $\operatorname{Tr}\left[X^{n} L^{m}\right]$. To calculate the time derivative, it is simplest to work with unrotated operators Eq. 29) and rotate back after differentiation. This yields

$$
\begin{aligned}
& \partial_{t} \operatorname{Tr}\left[(p-L)^{-1}(x-X)^{-1}\right]=\operatorname{Tr}\left[(p-L)^{-1} F(X)(p-L)^{-1}(x-X)^{-1}\right]+\operatorname{Tr}\left[(p-L)^{-1}(x-X)^{-1} L(x-X)^{-1}\right] \\
= & \operatorname{Tr}\left[(p-L)^{-2} F(X)(x-X)^{-1}\right]+\operatorname{Tr}\left[(p-L)^{-1} L(x-X)^{-2}\right]+\mathcal{O}([X, L]) \\
= & -\partial_{p} \operatorname{Tr}\left[(p-L)^{-1} F(X)(x-X)^{-1}\right]-\partial_{x} \operatorname{Tr}\left[(p-L)^{-1} L(x-X)^{-1}\right]+\mathcal{O}([X, L])
\end{aligned}
$$

In the second line, we invoked a semiclassical approximation consisting of ignoring the commutator $[X, L]$ (and, assuming $F$ is analytical, $[F(X), L])$. We shall discuss the physical interpretation and validity of this step below. 

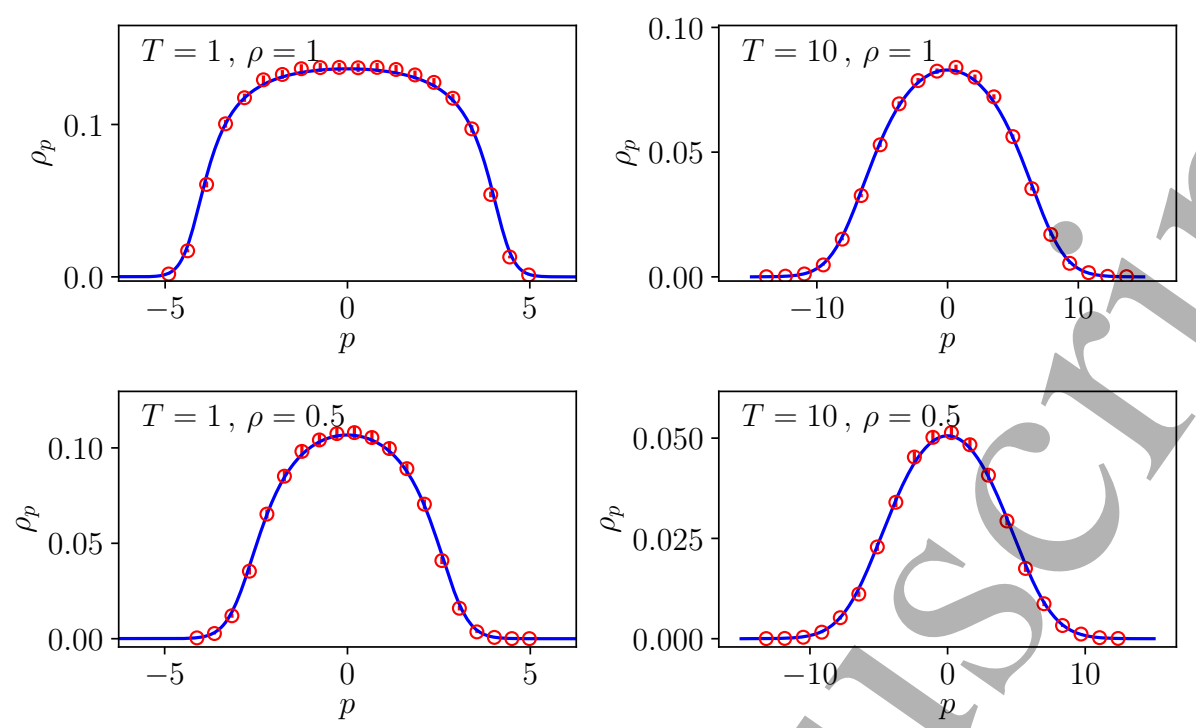

FIG. 1. Thermal quasiparticle density from the Lax matrix (markers) vs predictions from thermodynamic Bethe-Ansatz (TBA), Eqs. 190 and (22). For the Lax matrix data, we confine $N=256$ particles in a/box of length $L=N / \rho(\rho=1$ and $1 / 2)$ and generate $10^{4}$ thermal states of temperature $1 / \beta=1$ and 10 using the standard Markov-chain Monte Carlo method. We diagonalize the resulting Lax matrices and plot the averaged density of states (normalized so that $\int d p \rho_{p}=\rho$ ). To obtain the TBA prediction, we solve the Yang-Yang equation numerically and adjust the chemical potential $\mu^{c l}$ to match $\rho$. We set $\ell=1$ for all comparisons.

Assuming its validity for now and substituting into Eq. (46), we have

$$
\begin{aligned}
\partial_{t} \rho_{p}^{\mathrm{emp}}(x) & =-\partial_{p} \operatorname{Tr}[\delta(p-L) F(X) \delta(x-X)]-\partial_{x} \operatorname{Tr}[\delta(p-L) L \delta(x-X)]+\mathcal{O}([X, L]) \\
& =-\left(p \partial_{x}+F(x) \partial_{p}\right) \rho_{p}^{\mathrm{emp}}(x)+\mathcal{O}([X, L]) .
\end{aligned}
$$

We thus conclude that the empirical density satisfies the Boltzmann equation with the naïve forcing term, assuming the semiclassical approximation.

We now discuss the meaning of this approximation, and provide some arguments in its favour. First, we focus on the case without a trap, $F=0$ (the trapped case will be discussed in Section IV below). With no trap, we can show that this approximation captures the exact time evolution of the first and second moments in position of the empirical quasiparticle density. More concretely, the moments are defined as

$$
\left\langle x^{n}\right\rangle_{p}:=\int d x x^{n} \rho_{p}^{\mathrm{emp}}(x) .
$$

If $\rho_{p}^{\mathrm{emp}}(x)$ satisfies the non-interacting Boltzmann equation, we would expect $\frac{d}{d t}\left\langle x^{n}\right\rangle_{p}=p n\left\langle x^{n-1}\right\rangle_{p}$. Starting from the definition (44) and using the equations of motion Eqs. (33) and (34), it is not hard to check that this is indeed the case for $n \leq 2$ :

$$
\frac{d}{d t}\left\langle x^{n}\right\rangle_{p}=p n\left\langle x^{n-1}\right\rangle, n \leq 2, F=0 .
$$

In particular, $\partial_{t}\langle x\rangle_{p}=\rho_{p}^{J}$ is the current density, so the above identity for $n=1$ implies the current equation of state (27) for homogeneous states. Usual arguments then imply that the forceless Boltzmann equation Eq. (24) is satisfied if the empirical quasiparticle density varies slowly in space and time: the error term $\mathcal{O}([X, L])$ corresponds to subballistic corrections. To see this more explicitly, consider the $n=3$ moment (similar arguments apply for $n>3$, though their complication increases with $n$ ):

$$
\begin{aligned}
\partial_{t}\left\langle x^{3}\right\rangle_{p} & =3 p \mu_{2}+D_{2} \\
D_{2} & =\operatorname{Tr}[\delta(p-L)[X, L] X] \\
& =i \ell \sum_{j=1}^{N} \delta\left(p-\lambda_{j}\right)\left\langle\lambda_{j} \mid u\right\rangle\left\langle u|X| \lambda_{j}\right\rangle-i \ell\langle x\rangle_{p}
\end{aligned}
$$


where $|u\rangle=(1,1, \ldots, 1)^{T}$. On the right hand side of 51 , the ballistic term $\mu_{n-1}=\operatorname{Tr}\left[\delta(p-L) X^{n-1}\right]$, while the correction $D_{n}$ involve $(n-2)$ powers of $X$. Now, in a system with $N \gg 1$ particles at order 1 density, $\|X\| \sim N$ as an operator; hence prima facie, $D_{n}$ is of order $1 / N$ compared to the ballistic term. There is however a caveat: the vector $|u\rangle$ has norm $\sqrt{N}$. Nevertheless, its overlap with quasiparticle wavefunctions $\left|\left\langle u \mid \lambda_{j}\right\rangle\right|^{2}$ is large $(\sim N$ at most) only if the wavefunction is delocalized; for localized wavefunctions, the overlap is $1 / N$ smaller, of order unity. In the latter case, the term involving $|u\rangle$ is also subleading in the hydrodynamic limit.

Thus the semiclassical approximation relies on the localization of quasiparticle eigenstates. (This is after all expected, since by basic quantum mechanics $[X, L]$ measures the position uncertainty of the quasiparticle eigenstates.) Now, at nonzero temperature, the thermal ensemble of Lax matrices $L$ closely resembles a power-law random matrix ensemble studied recently $\sqrt{50}$, for which it is shown that most eigenstates are localized algebraically: $\left|\left\langle x_{a} \mid \lambda_{j}\right\rangle\right|^{2} \sim\left|x_{a}-x\right|^{-\gamma}$ for $\gamma \approx 2$, where $x$ is the localization centre of the wavefunction. On the other hand, as $T \rightarrow 0$, the randomness disappears and the quasiparticles delocalize. We therefore expect the most stringent dynamical tests of the Bethe-Lax correspondence to be quenches from zero/low temperature states, in presence of spatial inhomogeneity.

We now offer some more speculative remarks on the validity of the non-interacting Boltzmann description Eq. (48). First, notice that the commutator correction to the $n>2$ moment equations, as in Eq. (53), while subleading in $N$ also scales with the interaction strength $\ell$. Thus at the level of hydrodynamics, the Calogero interaction strength controls the strength of higher-order corrections to the non-interacting Boltzmann description. In the standard Calogero model, the interaction strength $\ell$ is taken to be independent of $N$. However, Eq. (53) suggests a possibility of accessing different dynamical scaling regimes by varying the scaling of $\ell$ with $N$.

We next note that although the empirical distribution function does not, in general, satisfy the non-interacting Boltzmann equation exactly, there is another distribution function,

$$
\rho_{p}^{\mathrm{ex}}(x)=\sum_{j=1}^{N} \delta\left(x-\langle X\rangle_{j}\right) \delta\left(p-\lambda_{j}\right)
$$

where $\langle X\rangle_{j}=\left\langle\lambda_{j}|X| \lambda_{j}\right\rangle$, which does satisfy Eq. (24) exactly when $F=0$, as a consequence of the microscopic equations of motion Eqs. (33) and (34). On the other hand, Eq. (54) is somewhat unphysical, in the sense that it is non-local in the bare particle coordinates $x_{a}$. Nevertheless, a comparison with the empirical density of states Eq. 43, together with the identity $\left|\left\langle x_{a} \mid \lambda_{j}\right\rangle\right|^{2}=\partial\langle X\rangle_{j} / \partial x_{a}$, suggests the identification

$$
\delta\left(x-\langle X\rangle_{j}\right) \approx \sum_{a=1}^{N} \frac{\partial\langle X\rangle_{j}}{\partial x_{a}} \delta\left(x-x_{a}\right) .
$$

This conjectural formula relates the local density of a single quasiparticle to the local densities of all bare particles. Its Jacobian form is reminiscent of the idea that quasiparticle dressing in integrable systems is equivalent to a nonlinear coordinate transformation from free particles $519151+53$. By the previous discussion, we expect that the error in Eq. is also controlled by the degree of quasiparticle localization, i.e. the magnitude of the commutator $[X, L]$. However, since Eq. (54) does not generalize readily beyond harmonic trapping potentials (unlike the more approximate, but more physical, empirical quasiparticle density), we shall not pursue its investigation further in this paper.

\section{E. Two-reservoir initial condition}

To conclude this section, we test the kinetic theory of $\rho_{p}^{\mathrm{emp}}(x)$ numerically in a standard "two-reservoir" setting. For this purpose, we prepare two different equilibria confined to the boxes $[-A, 0]$ and $[0, B]$ respectively (usually the reservoirs are considered semi-infinite; yet such a limit is hard to attain with the Calogero long-range interaction) . At $t=0$, the particles are released. We then measure the time evolution of particle and energy density under the Calogero dynamics (with $F=0$ ). We note that the long-range Calogero interaction means that an a priori definition of the energy density is not obvious. We define it using the Bethe-Lax correspondence, as

$$
\rho_{E}(x)=\int d p \frac{1}{2} p^{2} \rho_{p}^{\mathrm{emp}}(x)
$$

The numerical data are then compared with the non-interacting kinetic theory prediction

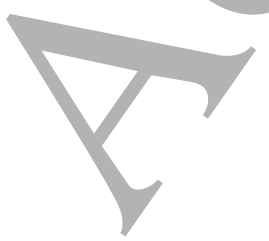

$$
\rho(x, t)=\int_{x / t}^{(x+A) / t} d p \rho_{L}(p)+\int_{(x-B) / t}^{x / t} d p \rho_{R}(p)
$$



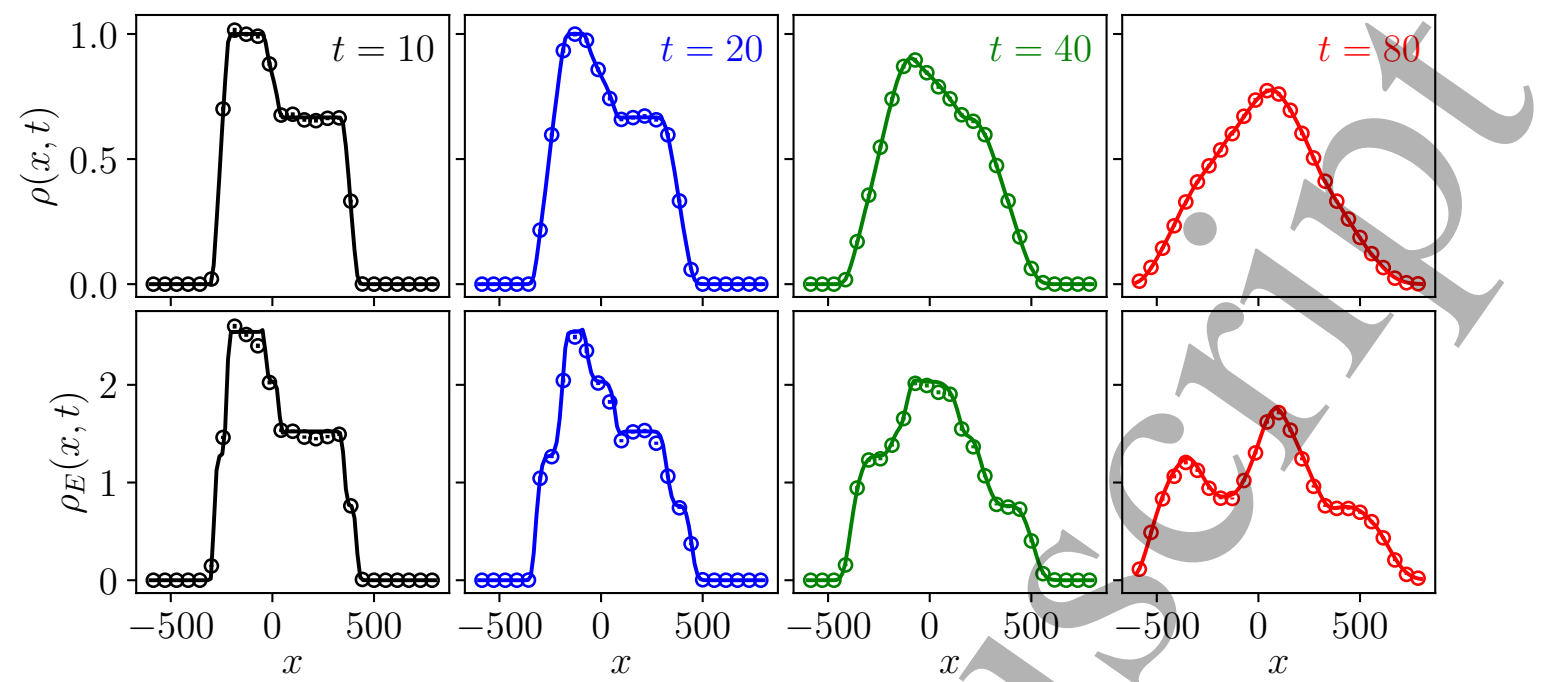

FIG. 2. Time evolution of particle and energy density starting from a two-reservoir initial condition. Left reservoir: $N=256$ particles confined to $[-N, 0]$ at $T=1$. Right reservoir: 256 particles confined to $[0,3 N / 2]$ at $T=2$. The numerical data (circles) is averaged over 400 thermal samples. The analytical prediction (solid curves) is obtained from the TBA equation and the free Boltzmann equation, see Eq. (57). We set $\ell=1$ for all plots.

where $\rho_{L}$ and $\rho_{R}$ are the quasiparticle density of the reservoirs, obtained by TBA. An excellent agreement is found, see Fig. 2. This is not trivial given that the temperatures of the reservoirs are low enough that the localization length of the quasiparticle wavefunctions (at $t=0$ ) is about half the box size. Even so, the semiclassical approximation appears to be robust.

\section{TRAPPED DYNAMICS}

We argued above that the quasiparticle density of the Calogero model, defined via the Bethe-Lax correspondence, satisfies a non-interacting Boltzmann equation even in the presence of trapping potentials. In this section, we test the non-interacting Boltzmann description against/microscopic simulations of dynamics in harmonic and anharmonic traps. We find excellent agreement in all cases, even for complicated nonlinear phenomena such as soliton dynamics in the harmonically trapped Calogero-Moser mode ${ }^{30}$.

\section{A. Harmonic trap}

As recalled in Section IIB the classical Calogero model remains integrable in the presence of a harmonic trapping potential, $V(x)=\frac{1}{2} \omega^{2} x^{2}$. In the matrix model formulation Eq. 29, the corresponding potential reads $V(M)=$ $\frac{1}{2} \omega^{2} M^{2}$. Thus $F(M)=-\omega^{2} M$ and the variable

simply rotates:

$$
Q=\omega M-i \dot{M},
$$

$$
\dot{Q}=i \omega Q \Rightarrow Q(t)=e^{i \omega t} Q(0) .
$$

From periodicity of $Q$, it is immediate that the dynamics of the harmonically trapped Calogero model exhibits "perfect revivals" at each trap period, $T=2 \pi / \omega$.

Intriguingly, this property is shared by the naïve kinetic theory Eq. 25, which for a harmonic trap takes the form

$$
\partial_{t} \widetilde{\rho}_{p}+p \partial_{x} \widetilde{\rho}_{p}-\omega x \partial_{p} \widetilde{\rho}_{p}=0
$$




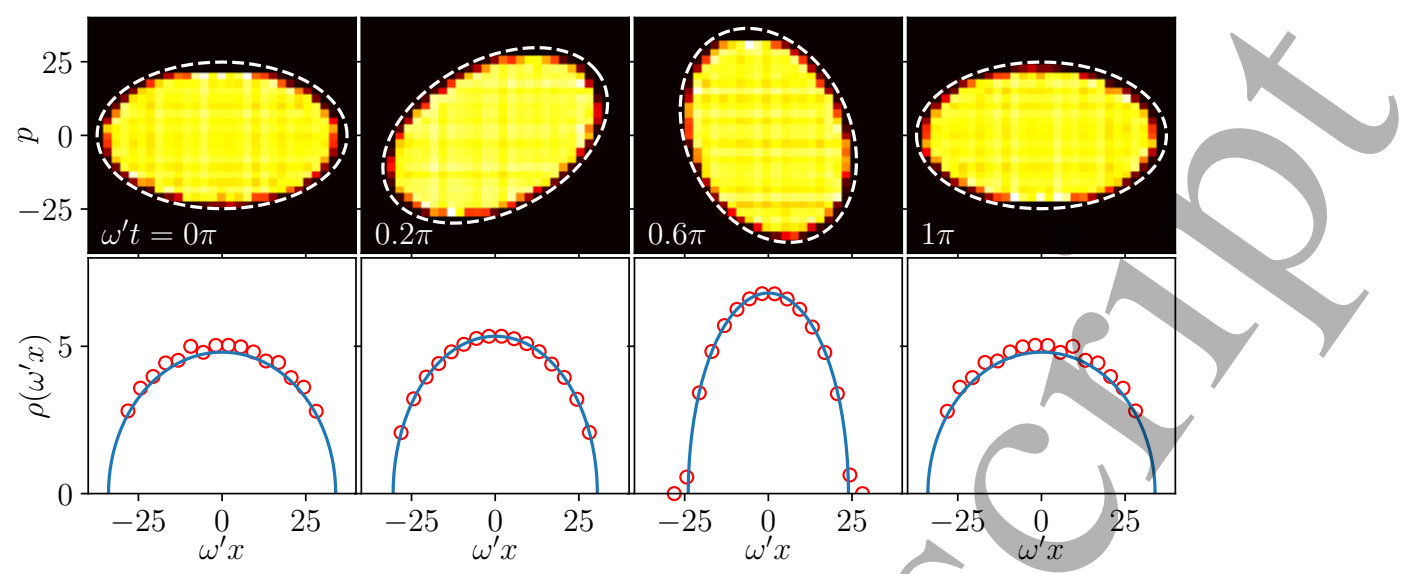

FIG. 3. Top panels: the empirical quasiparticle density in a harmonic trap with frequency $\omega^{\prime}=1.5$, evolving from an initial condition consisting of a thermal cloud of 256 particles at temperature $T=1$ and confined to a harmonic trap with frequency $\omega=1$. The dashed curves show an ellipse that rotates with angular velocity $\omega^{\prime}$. The bottom panels compare the measured particle density (circles, average of 200 thermal samples) to the naïve kinetic theory prediction (solid curves). We set $\ell=1$ for all plots.

and has an exact solution by characteristics, with characteristic curves given by

$$
\frac{d}{d t} x=p, \quad \frac{d}{d t} p=-\omega x,
$$

corresponding to uniform rotation of the distribution function $\rho$ with frequency $\omega$ on constant-energy ellipses, $p^{2}+$ $\omega^{2} x^{2}=2 E$.

The Bethe-Lax correspondence provides a direct relation between the Boltzmann equation Eq. $(60)$ and the microscopic motion. Indeed, it predicts that the empirical quasiparticle density $\rho^{\mathrm{emp}}$ rotates in the simple manner described above. In Section IIID, we showed that $\rho^{\mathrm{emp}}$ satisfies Eq 600 under the semiclassical approximation but only discussed its justification in the case $F=0$. For a harmonic trap $F=\omega X$, the situation is similar. The naïve kinetic theory (60) again yields the exact time evolution of the lowest moments, but one must now consider moments in both $x$ and $p$. Indeed, defining

$$
\left\langle x^{n} p^{m}\right\rangle:=\int d x d p x^{n} p^{m} \rho_{p}^{\mathrm{emp}}(x)=\operatorname{Tr}\left[X^{n} L^{m}\right],
$$

the equations of motion (33), (34) imply that

$$
\frac{d}{d t}\left\langle x^{n} p^{m}\right\rangle \triangleq n\left\langle x^{n-1} p^{m+1}\right\rangle-m \omega\left\langle x^{n+1} p^{m-1}\right\rangle, n \leq 2, m \leq 2,
$$

which can also be derived by assuming $\rho_{p}^{\mathrm{emp}}(x)$ satisfies 60 . For higher moments, the above equation still holds modulo a correction which has a commutator $[X, L]$ and $n+m-1$ powers of $X$ and $L$, which is one less compared to the RHS of 63). The argument then proceeds as in the flat case, provided one observes that in presence of the trap, both $X$ and $L$ have eigenvalues of order $\sqrt{N}$ typically (recall that in contrast, $L \sim \mathcal{O}(1)$ and $X \sim \mathcal{O}(N)$ typically in the flat case). Hence we expect finite-size effects to be more pronounced in the presence of a trapping potential.

We now test and illustrate the Bethe-Lax correspondence with two numerical examples. First, we consider a quench in which a Calogero-Moser gas is prepared at low temperature $T$ in a trap of frequency $\omega$, and at $t=0$, the trap frequency is quenched to $\omega^{\prime} \neq \omega$. We then compute the empirical quasiparticle density at different times (by solving the dynamics using Eq. (59) and diagonalizing the Lax matrix). Plotting this in the $\left(\omega^{\prime} x, p\right)$ phase space, we can clearly see that the empirical quasiparticle density rotates simply as predicted by the naïve kinetic theory, see Fig. 3 . We also verify that this picture yields quantitatively correct predictions for the local particle density, despite the pronounced finite-size effect (in particular near the edge of the distribution).

As a more spectacular example, we consider a two-soliton solution, revealed in Ref. 30. The initial conditions for the two soliton solution are chosen as follows. We choose two dual complex variables $z_{1}$ and $z_{2}$. The initial conditions 

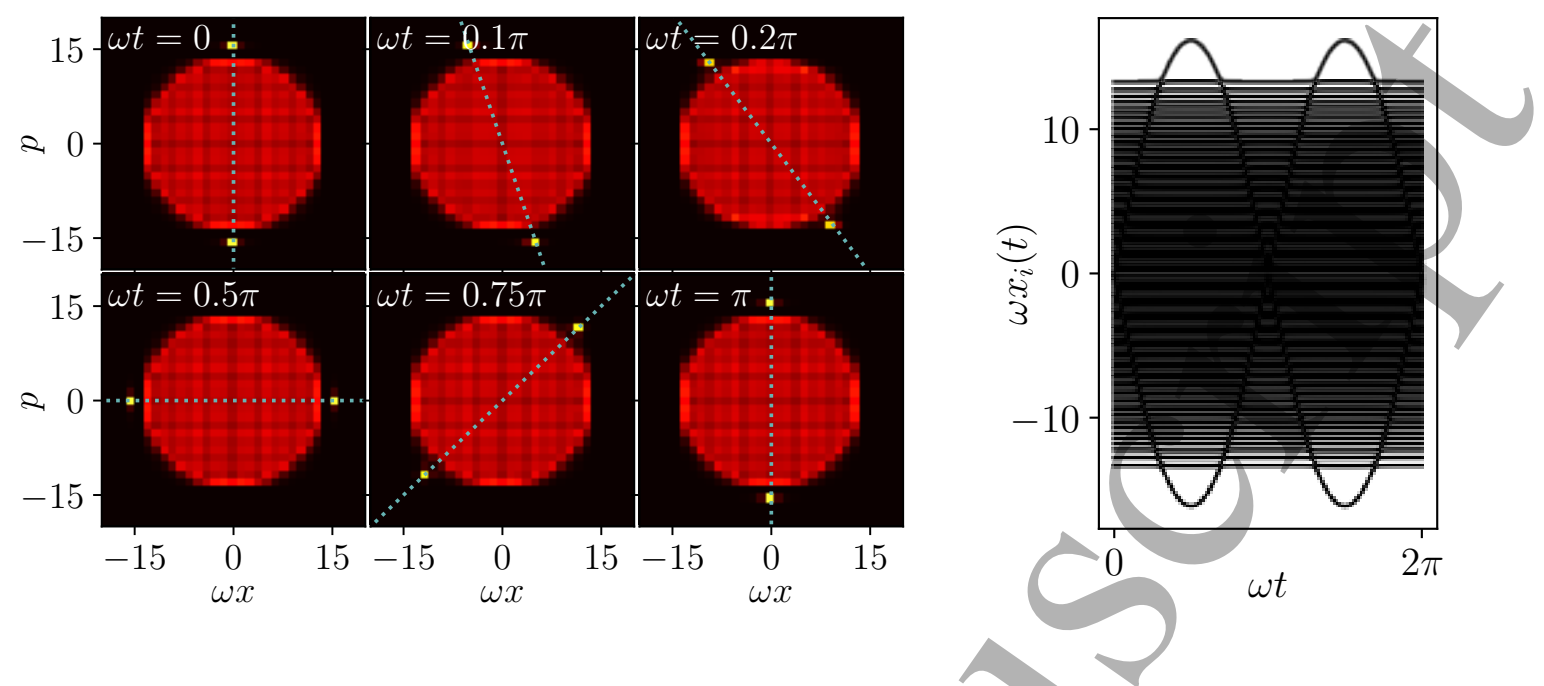

FIG. 4. Left panels: Time evolution of the empirical quasiparticle density of a two-soliton solution (64), 65, with $\ell=1$, $\omega=1, z_{1}=-z_{2}=0.251247 i, N=101$. The dashed line rotates with angular velocity $\omega$. The solitons appear as peaks in the $(x, p)$ plane, rotating with angular velocity $\omega$. Right panel: the evolution of particle positions of the same solution, over a period. Note that the trajectories do not cross each other; the solitons are a collective phenomenon involving all particles.

are chosen such that the following conditions are satisfied:

$$
\begin{aligned}
\omega x_{j} & =\ell \sum_{k=1(k \neq j)}^{N} \frac{1}{x_{j}-x_{k}}-\frac{\ell}{2}\left(\frac{1}{x_{j}-z_{1}}+\frac{1}{x_{j}-\bar{z}_{1}}+\frac{1}{x_{j}-z_{2}}+\frac{1}{x_{j}-\bar{z}_{2}}\right) \\
p_{j} & =\frac{i \ell}{2}\left(\frac{1}{x_{j}-z_{1}}-\frac{1}{x_{j}-\bar{z}_{1}}+\frac{1}{x_{j}-z_{2}}-\frac{1}{x_{j}-\bar{z}_{2}}\right)
\end{aligned}
$$

For fixed $z_{1}$ and $z_{2}$, Eq. (64) and Eq. 65) are non-trivial to solve. However, one can employ a damping equation 2654 that leads to a potential minimization problem. Once we find the initial condition for the two-soliton case, the subsequent evolution can be performed via the matrix formulation given in Eq. (59). We then numerically compute the empirical quasiparticle density of the two-soliton solution and plot it in Fig. 4 . Remarkably, the solitons manifest themselves as sharp peaks on either side of the disk distribution (which corresponds to the zero-temperature state in a harmonic trap). The peaks appear near the edge of the disk and rotate in phase space as if they were free particles in the harmonic potential, never encountering one another.

\section{B. Nonintegrable anharmonic traps}

A distinctive feature of the conjectured Bethe-Lax correspondence is that it can be formulated for general anharmonic traps, even non-integrable ones: the empirical quasiparticle density, defined in Eq. (44), should satisfy the non-interacting Boltzmann equation Eq. 25). By the arguments in Section IIID this statement is true under the "semiclassical approximation" in which commutators of the form $\mathcal{O}([X, L])$ are neglected. However, the dynamics of $X$ and $L$ depend on the shape of the potential under consideration, in such a way that it is difficult to formulate general analytical arguments beyond the harmonic case.

We therefore proceed directly to a numerical test. To this end, we prepare a zero- $T$ cloud in a harmonic potential $V(x)=\frac{1}{2} x^{2}$ and then quench to an anharmonic potential, $V(x)=2 \sqrt{1+x^{2}}$, that is not expected to preserve integrability. We see that the non-interacting Boltzmann equation Eq. (25) successfully captures the relaxation dynamics, which therefore consists solely of single-(quasi)particle dephasing on accessible time-scales.

The performance of the simple kinetic theory in an integrability-breaking trap is intriguing. On general grounds, we expect the agreement to break down eventually as the dynamics becomes chaotic 21 . However, for the trapped Calogero model we were unable to observe the onset of chaos on accessible time-scales. This is probably due to the robustness of the Calogero model's integrability to fourth-order trapping potentials (cf. the discussion around Eq. (26)), which suggests a robustness to trapping potentials in general, compared to other integrable models. This expectation is borne out by numerical simulations of Poincaré sections for the two-particle Calogero model in an 


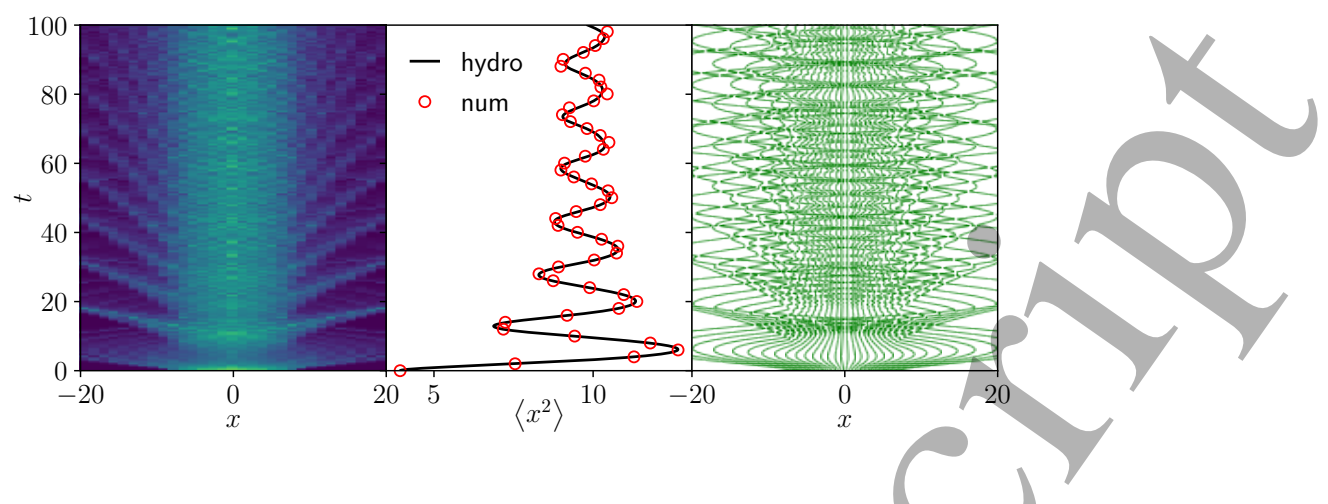

FIG. 5. Left: non-interacting Boltzmann prediction for the particle density. Middle: comparison between hydrodynamic and microscopic predictions for the mean squared width of the packet. Right: microscopic evolution. Model parameters are set to $N=32, \ell=1$.
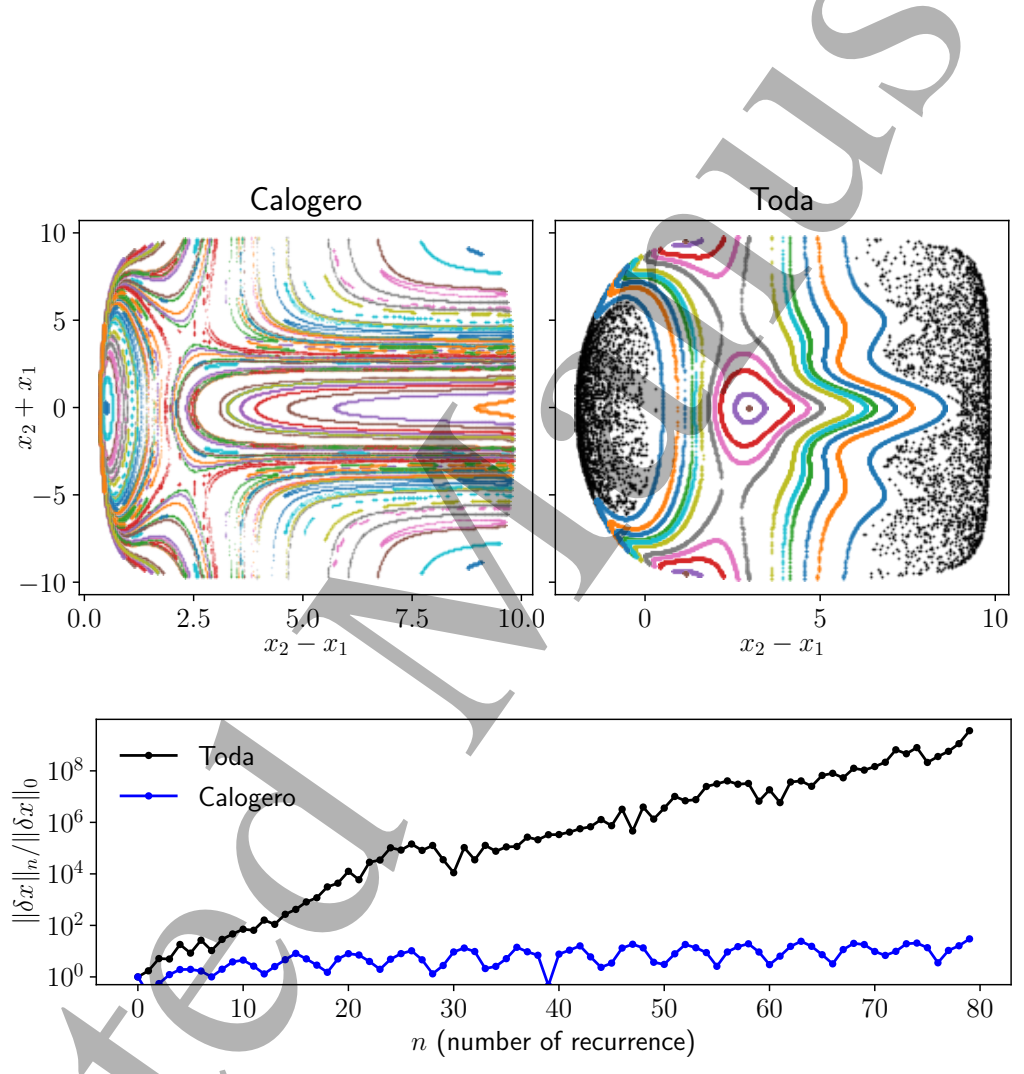

FIG. 6. Top panels: Orbits of the recurrence map in the Poincaré section defined by $E=10$ and $p_{1}=-p_{2}$, in a system with two particles in an external potential $V(x)=\sqrt{x^{2}+1}$ and interacting with potentials $1 /\left(x_{1}-x_{2}\right)^{2}(\mathrm{Calogero}$, left $)$ and $e^{-\left(x_{1}-x_{2}\right)}$ (Toda, right). Different colors are assigned randomly to trajectories to distinguish them. Integrability of the Toda interaction is broken by the trap: the black points represent a single chaotic trajectory that is ergodic in a large portion of the phase space. Meanwhile, that of the Calogero interaction seems robust. Bottom panel: Growth of the separation $\|\delta x\|_{n}=$ $\left|x_{1}(n)-x_{1}^{\prime}(n)\right|+\left|x_{2}(n)-x_{2}^{\prime}(n)\right|$ between a pair of nearby orbits $\left(x_{i}\right.$ and $x_{i}^{\prime}$, separated by an initial distance $\left.\|\delta x\|_{0}=10^{-8}\right)$ under either dynamics. In the Toda case, the orbits start from $x_{1}(0)+x_{2}(0)=0.1, x_{1}(0)-x_{2}(0)=-0.5$ in the chaotic basin, and exhibit exponential growth with a positive Lyapuov exponent. In the Calogero case, we found no such chaotic trajectory after an exhaustive search. The illustrated orbit starts from $x_{1}(0)+x_{2}(0)=0.1, x_{1}(0)-x_{2}(0)=3$.

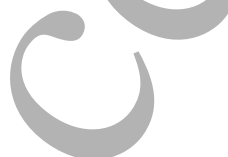

integrability-breaking trap compared to the two-particle Toda chain in such a trap, see Fig. 6. It is also consistent with studies of the Toda chain in power-law pinning potentials 55 , which exhibit a crossover to normal diffusion at large system sizes, at variance with our observations of purely ballistic evolution for the Calogero model. 


\section{DISCUSSION}

We have derived the kinetic theory of Calogero quasiparticles on a line, which reduces to the non-interacting Boltzmann equation. We showed that this simplification could be understood from an emergent quasiparticle description in terms of eigenvectors of the Lax matrix. The resulting expression for the empirical single-particle distribution function, Eq. (43), yields excellent agreement with the non-interacting Boltzmann equation on accessible time-scales, even in the presence of integrability-breaking trapping potentials.

One point which merits further comment is the long-ranged character of interactions in the Calogero model. At first sight, such long-ranged interactions render the conserved charges and currents of the Calogero model non-local $\underline{45}$, which calls into question the validity of a local, hydrodynamic description. At the same time, the Calogero model is exactly solvable using the asymptotic Bethe ansat ${ }^{57}$, suggesting that the scattering behaviour of its quasiparticles is much the same as for short-range-interacting integrable models. Indeed, the validity of hydrodynamics for the Calogero model is most easily justified in the quasiparticle language: according to the Bethe-Lax correspondence presented above, dynamics in the Calogero model is captured by quasiparticles that are localized in space at nonzero temperature, despite the long-ranged interactions between bare particles. In this sense, the quasiparticle kinetic theory of the Calogero model is no different from that of a short-ranged integrable model.

We noted earlier that there is a mature theory of the zero-temperature ("superfluid") hydrodynamics of quantum and classical Calogero models based on collective field theory 27 . When subballistic derivative corrections to the latter are neglected, it matches the zero-temperature limit of our results. We anticipate that a systematic treatment of the commutator corrections to the free Boltzmann evolution in Eq. (48) (or perhaps a first-principles treatment of Eq. (55)) would recover these subballistic derivative corrections at non-zero temperature. Another interesting common feature between our analysis and the collective field theory of Calogero models is that the latter remains integrable in arbitrary external trapping potentials 58 , just like the non-interacting Boltzmann equation Eq. 25]. Finally, a natural extension of our work would be to develop a kinetic theory for the broader family of compactified Calogero-Sutherland models with trigonometric or hyperbolic interactions, which inherit the property of remaining integrable in suitably chosen trapping potentials ${ }^{26}$. It seems plausible that a Bethe-Lax correspondence analogous to Eqs. (44) and (45) holds for all of these models.

Our results demonstrate that the integrability-breaking effects of external trapping potentials, as arise in presentday experiments on ultracold Bose gases $\frac{17|19| 59}{\text {, }}$, can be rather subtle. For example, the absence of diffusion on the Navier-Stokes scale for both the quantum and classical Calogero models seems to imply that the mechanism of diffusive thermalization proposed recently for trapped integrable gases ${ }^{2325}$ does not apply. A related question is whether the trapped Calogero model exhibits a finite "time to chaos", that was observed for systems of trapped classical hard rods ${ }^{21}$ but appears to be numerically inaccessible for the Calogero model, even in traps that are expected to break integrability. Both findings suggest that the tendency to chaos for trapped Calogero particles, quantum or classical, is remarkably weak. Thus identifying the signatures of broken integrability in the Calogero model might pave the way towards a deeper understanding of broken integrability in the quantum Newton's cradle 5 .

Acknowledgments. We are grateful to A.G. Abanov for helpful discussions and to H. Spohn for collaborations on related topics. We thank S. Santra for help with data for thermal initial conditions for the trapped case. MK would like to acknowledge support from the project 6004-1 of the Indo-French Centre for the Promotion of Advanced Research (IFCPAR), Ramanujan Fellowship (SB/S2/RJN-114/2016), SERB Early Career Research Award (ECR/2018/002085) and SERB Matrics Grant (MTR/2019/001101) from the Science and Engineering Research Board (SERB), Department of Science and Technology, Government of India. MK acknowledges support of the Department of Atomic Energy, Government of India, under Project No. RTI4001. J.E.M. acknowledges support from US National Science Foundation grant DMR-1918065.

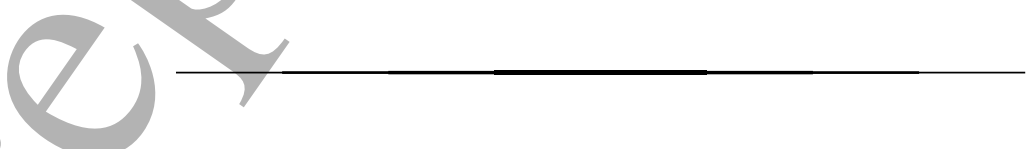

[1] H. Spohn, Large scale dynamics of interacting particles (Springer Science \& Business Media, 2012).

[2] L. Erdős, B. Schlein, and H.-T. Yau, Derivation of the Gross-Pitaevskii equation for the dynamics of Bose-Einstein condensate, Annals of mathematics , 291 (2010).

[3] O. A. Castro-Alvaredo, B. Doyon, and T. Yoshimura, Emergent Hydrodynamics in Integrable Quantum Systems Out of Equilibrium, Physical Review X 6, 041065 (2016), arXiv:1605.07331.

[4] B. Bertini, M. Collura, J. De Nardis, and M. Fagotti, Transport in Out-of-Equilibrium XXZ Chains: Exact Profiles of Charges and Currents., Physical review letters 117, 207201 (2016), arXiv:1605.09790.

[5] J. K. Percus, Exact solution of kinetics of a model classical fluid, The Physics of Fluids 12, 1560 (1969), http://aip.scitation.org/doi/pdf/10.1063/1.1692711

[6] V. Zakharov, Kinetic equation for solitons, Sov. Phys. JETP 33, 538 (1971). 
[7] C. Boldrighini, R. L. Dobrushin, and Y. M. Sukhov, One-dimensional hard rod caricature of hydrodynamics, Journal of Statistical Physics 31, 577 (1983).

[8] G. A. El and A. M. Kamchatnov, Kinetic Equation for a Dense Soliton Gas, Phys. Rev. Lett. 95, 204101 (2005).

[9] B. Doyon, T. Yoshimura, and J.-S. Caux, Soliton gases and generalized hydrodynamics, Phys. Rev. Lett. 120, 045301 $(2018)$

[10] V. B. Bulchandani, R. Vasseur, C. Karrasch, and J. E. Moore, Bethe-Boltzmann hydrodynamics and spin transport in the XXZ chain, Physical Review B 97, 10.1103/PhysRevB.97.045407 (2018), arXiv:1702.06146

[11] S. Gopalakrishnan, D. A. Huse, V. Khemani, and R. Vasseur, Hydrodynamics of operator spreading and quasiparticle diffusion in interacting integrable systems, Phys. Rev. B 98, 220303 (2018)

[12] S. Gopalakrishnan and R. Vasseur, Kinetic Theory of Spin Diffusion and Superdiffusion in $X X Z$ Spin Cháins, Phys. Rev. Lett. 122, $127202(2019)$.

[13] A. C. Cubero and M. Panfil, Generalized hydrodynamics regime from the thermodynamic bootstrap program, SciPost Phys. 8, 4 (2020)

[14] M. Fagotti, Locally quasi-stationary states in noninteracting spin chains, SciPost Phys. 8, 48 (2020)

[15] T. Yoshimura and H. Spohn, Collision rate ansatz for quantum integrable systems, SciPost Phys. 9, 40 (2020)

[16] B. Pozsgay, Algebraic construction of current operators in integrable spin chains, Phys. Rev. Lett. 125, 070602 (2020)

[17] M. Schemmer, I. Bouchoule, B. Doyon, and J. Dubail, Generalized hydrodynamics on an atom chip, Phys. Rev. Lett. 122, 090601 (2019)

[18] A. Scheie, N. E. Sherman, M. Dupont, S. E. Nagler, M. B. Stone, G. E. Granroth, J. E. Moore, and D. A. Tennant, Detection of Kardar-Parisi-Zhang hydrodynamics in a quantum Heisenberg spin-1/2 chain, Nature Physics 10.1038/s41567-02101191-6 (2021).

[19] N. Malvania, Y. Zhang, Y. Le, J. Dubail, M. Rigol, and D. S. Weiss, Generalized hydrodynamics in strongly interacting 1d bose gases (2020), arXiv:2009.06651 [cond-mat.quant-gas]

[20] B. Doyon and T. Yoshimura, A note on generalized hydrodynamics: inhomogeneous fields and other concepts, SciPost Phys. 2, 014 (2017)

[21] X. Cao, V. B. Bulchandani, and J. E. Moore, Incomplete thermalization from trap-induced integrability breaking: Lessons from classical hard rods, Phys. Rev. Lett. 120, 164101 (2018)

[22] J.-S. Caux, B. Doyon, J. Dubail, R. Konik, and T. Yoshimura, Hydrodynamics of the interacting Bose gas in the Quantum Newton Cradle setup, SciPost Phys. 6, 70 (2019)

[23] A. Bastianello, A. De Luca, B. Doyon, and J. De Nardis, Thermalization of a Trapped One-Dimensional Bose Gas via Diffusion, Phys. Rev. Lett. 125, 240604 (2020)

[24] A. Bastianello, A. D. Luca, and R. Vasseur, Hydrodynamics of weak integrability breaking (2021), arXiv:2103.11997 [cond-mat.stat-mech]

[25] J. Durnin, A. D. Luca, J. D. Nardis, and B. Doyon, Diffusive hydrodynamics of inhomogenous hamiltonians (2021), arXiv:2105.13068 [cond-mat.stat-mech].

[26] M. Kulkarni and A. Polychronakos, Emergence of the calogero family of models in external potentials: duality, solitons and hydrodynamics, Journal of Physics A: Mathematical and Theoretical 50, 455202 (2017).

[27] A. G. Abanov and P. B. Wiegmann, Quantum hydrodynamics, the quantum benjamin-ono equation, and the calogero model, Phys. Rev. Lett. 95, $076402(2005)$

[28] A. G. Abanov, E. Bettelheim, and P. Wiegmann, Integrable hydrodynamics of calogero-sutherland model: bidirectional benjamin-ono equation, Journal of Physics A: Mathematical and Theoretical 42, 135201 (2009).

[29] M. Stone, I. Anduaga, and L. Xing, The classical hydrodynamics of the Calogero-Sutherland model, Journal of Physics A: Mathematical and Theoretical 41, 275401 (2008).

[30] A. G. Abanov, A. Gromov, and M. Kulkarni, Soliton solutions of a calogero model in a harmonic potential, Journal of Physics A: Mathematical and Theoretical 44, 295203 (2011).

[31] V. B. Bulchandani, X. Cao, and J. E. Moore, Kinetic theory of quantum and classical toda lattices, Journal of Physics A: Mathematical and Theoretical 52, 33LT01 (2019).

[32] N. Theodorakopoulos, Finite-Temperature Excitations of the Classical Toda Chain, Phys. Rev. Lett. 53, 871 (1984).

[33] P. Gruner-Bauer and F. G. Mertens, Excitation spectrum of the Toda lattice for finite temperatures, Zeitschrift für Physik B Condensed Matter 70, 435 (1988)

[34] A. Bastianello, B. Doyon, G. Watts, and T. Yoshimura, Generalized hydrodynamics of classical integrable field theory: the sinh-Gordon model, SciPôst Phys. 4, 45 (2018)

[35] H. Spohn, Generalized Gibbs Ensembles of the Classical Toda Chain, Journal of Statistical Physics 180, 4-22 (2019).

[36] B. Doyon, Generalized hydrodynamics of the classical toda system, Journal of Mathematical Physics 60, 073302 (2019), https://doi.org/10.1063/1.5096892.

[37] X. Cao, V. B. Bulchandani, and H. Spohn, The GGE averaged currents of the classical toda chain, Journal of Physics A: Mathematical and Theoretical 52, 495003 (2019).

[38] H. Spohn, Hydrodynamic Equations for the Toda Lattice (2021), arXiv:2101.06528 [cond-mat.stat-mech].

[39] B. Sutherland, Quantum many-body problem in one dimension: Thermodynamics, Journal of Mathematical Physics 12, 251 (1971), https://doi.org/10.1063/1.1665585.

[40] C. N. Yang and C. P. Yang, Thermodynamics of a one-dimensional system of bosons with repulsive delta-function interaction, Journal of Mathematical Physics 10, 1115 (1969), https://doi.org/10.1063/1.1664947.

[41] S. B. Isakov, Fractional statistics in one dimension: Modeling by means of $1 / x^{2}$ interaction and statistical mechanics, International Journal of Modern Physics A 09, 2563 (1994), https://doi.org/10.1142/S0217751X94001023 
[42] V. B. Bulchandani, S. Gopalakrishnan, and E. Ilievski, Superdiffusion in spin chains (2021), arXiv:2103.01976 [condmat.stat-mech].

[43] J. De Nardis, D. Bernard, and B. Doyon, Hydrodynamic Diffusion in Integrable Systems, Physical Review Letters 121, 10.1103/PhysRevLett.121.160603 (2018), arXiv:1807.02414.

[44] S. Gopalakrishnan, D. A. Huse, V. Khemani, and R. Vasseur, Hydrodynamics of operator spreading and quasiparticle diffusion in interacting integrable systems, Physical Review B 98, 10.1103/PhysRevB.98.220303 (2018), arXiv:1809.02126.

[45] H. Spohn, Interacting and noninteracting integrable systems, Journal of Mathematical Physics 59, 091402 (2018), https://doi.org/10.1063/1.5018624.

[46] P. Choquard, Classical and Quantum Partition Functions of the Calogero-Moser-Sutherland Model, in Calogero-MoserSutherland Models, edited by J. F. van Diejen and L. Vinet (Springer New York, New York, NY, 2000) pp. 117-125.

[47] F. Calogero, Solution of the one-dimensional n-body problems with quadratic and/or inversely quadratic pair potentials, Journal of Mathematical Physics 12, 419 (1971), https://doi.org/10.1063/1.1665604.

[48] A. P. Polychronakos, The physics and mathematics of calogero particles, Journal of Physics A: Mathematical and General 39, 12793 (2006).

[49] H. Flaschka, The Toda lattice. II. Existence of integrals, Phys. Rev. B 9, 1924 (1974)

[50] X. Deng, V. E. Kravtsov, G. V. Shlyapnikov, and L. Santos, Duality in power-law localization in disordered one-dimensional systems, Phys. Rev. Lett. 120, 110602 (2018).

[51] B. Doyon, H. Spohn, and T. Yoshimura, A geometric viewpoint on generalized hydrodynamics, Nuclear Physics B 926, $570(2018)$

[52] V. B. Bulchandani, On classical integrability of the hydrodynamics of quantum integrable systems, Journal of Physics A: Mathematical and Theoretical 50, 435203 (2017).

[53] J. Cardy and B. Doyon, TT deformations and the width of fundamental particles (2021), arXiv:2010.15733 [hep-th].

[54] A. K. Gon and M. Kulkarni, Duality in a hyperbolic interaction model integrable even in a strong confinement: multi-soliton solutions and field theory, Journal of Physics A: Mathematical and Theoretical 52, 415201 (2019).

[55] P. Di Cintio, S. Iubini, S. Lepri, and R. Livi, Transport in perturbed classical integrable systems: The pinned toda chain, Chaos, Solitons and Fractals 117, 249-254 (2018)

[56] A. Dhar, A. Kundu, J. L. Lebowitz, and J. A. Scaramazza, Transport Properties of the Classical Toda Chain: Effect of a Pinning Potential, Journal of Statistical Physics 175, 1298-1310 (2019).

[57] B. Sutherland, Exact results for a quantum many-body problem in one dimension. ii, Phys. Rev. A 5, 1372 (1972)

[58] A. P. Polychronakos, A new integrable system with a quartic potential, Physics Letters B 276, 341 (1992).

[59] T. Kinoshita, T. Wenger, and D. S. Weiss, A quantum Newton's cradle, Nature 440, 900 EP (2006)
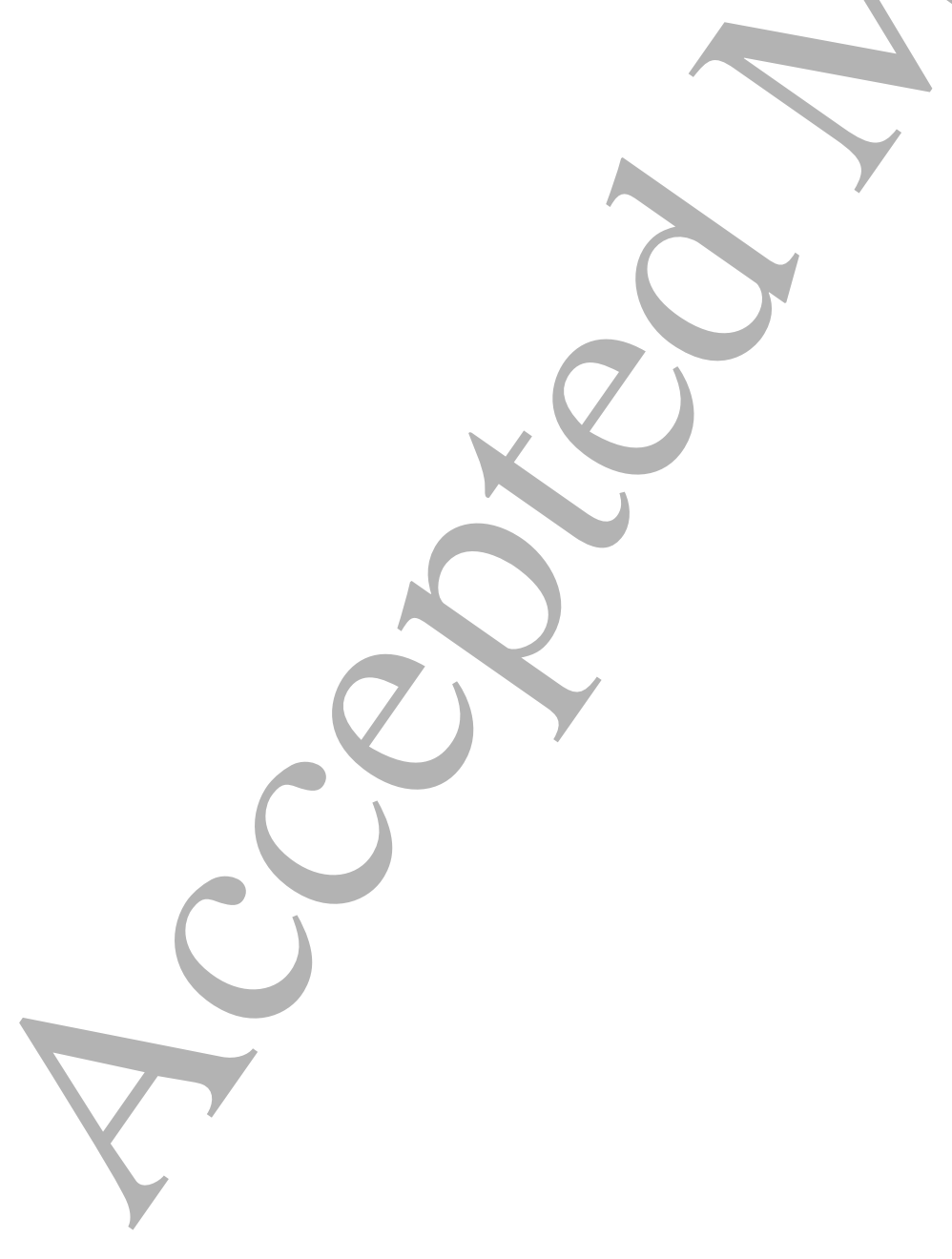